\begin{tabular}{llllll}
\multicolumn{5}{l}{ Abstract P066 Table 1} \\
\hline Chr & Coordinate & Gene & PSC & lgG4-SC & UC \\
\hline 5 & 93905427 & C5orf36 & $7.02 \times 10-^{17}$ & $4.45 \times 10-^{12}$ & $7.32 \times 10-^{17}$ \\
3 & 101443535 & CEP97 & $6.66 \times 10-^{15}$ & $2.62 \times 10-^{12}$ & - \\
17 & 7481193 & EIF4A1; & $8.48 \times 10-^{13}$ & - & $1.44 \times 10-^{9}$ \\
& & SNORA67 & & & \\
3 & 101443691 & CEP97 & $4.54 \times 10-^{11}$ & - & - \\
4 & 89300650 & HERC6 & $8.17 \times 10-^{11}$ & $4.37 \times 10-^{-9}$ & - \\
17 & 42075116 & PYY & $3.25 \times 10-^{10}$ & $7.8 \times 10-^{10}$ & $1.64 \times 10-^{14}$ \\
19 & 797342 & PTBP1 & $7.78 \times 10-^{10}$ & - & $6.16 \times 10-^{14}$ \\
4 & 48136298 & TXK & $2.89 \times 10-^{9}$ & - & - \\
3 & 96338474 & & $4.97 \times 10-^{9}$ & $2.59 \times 10-^{8}$ & - \\
8 & 125313885 & & $6.94 \times 10-^{9}$ & $3.85 \times 10-^{8}$ & - \\
4 & 48136234 & TXK & $6.34 \times 10-^{8}$ & - & $4.91 \times 10-^{8}$ \\
21 & 34696571 & IFNAR1 & - & $3.19 \times 10-^{10}$ & - \\
3 & 169489583 & MYNN & - & $1.13 \times 10-^{8}$ & - \\
\hline
\end{tabular}

in PSC-UC (6 not found in combined PSC) and 4 significant probes in PSC without IBD, of which only one was shared with PSC-UC. Analysis of the epigenetic clock did not show any age acceleration in PSC or IgG4-SC compared to healthy controls. Genotyping data revealed a strong signal in the HLA region in PSC, not observed in the other groups, though no findings were significant genome-wide as this study was underpowered as a GWAS. However, meQTLs in PSC were also strong in the HLA region with 19/40 significant findings in 4 clusters (HLA-DRB1, BTNL2, CSNK2B, and SFTA2). There was no overlap with the 36 significant meQTLs found in IgG4-SC. Of the significant disease-associated methylation differences PYY, involved in gut mobility and pancreatic secretion, and Interferon receptor subunit IFNAR1 stand out as targets for further investigation.

This study provides a novel description of whole-blood methylomes in PSC and IgG4-SC, and correlations with genetic variants. Future studies are needed to establish relationships with gene expression, and outcome measures.
Selected significant disease-associated methylation changes in PSC, IgG4-SC, and UC. P values are unadjusted, - denotes non-significance after Holm correction for multiple testing.

\section{P067 OUTCOMES IN PATIENTS WITH ACUTE LIVER FAILURE TREATED WITH LOW VOLUME PLASMA EXCHANGE: UK MULTICENTRE EXPERIENCE}

${ }^{1}$ Laura Burke*, ${ }^{2}$ Tasneem Pirani, ${ }^{2}$ Vadivukkarasi Thangara Jayalakshmi, ${ }^{3}$ Banwari Agarwal, ${ }^{3}$ Jennifer Ryan, ${ }^{4}$ Mansoor Banghash, ${ }^{4}$ Nicholas Murphy, ${ }^{4}$ Phil El-Dalil, ${ }^{5}$ Steve Masson, ${ }^{5}$ Mhairi Donnelly, ${ }^{2}$ William Bernal, ${ }^{1}$ Joanna K Moore. 'Leeds Teaching Hospitals NHS Trust, Leeds, UK; ${ }^{2}$ King's College Hospital NHS Foundation Trust, London, UK; ${ }^{3}$ Royal Free London NHS Foundation Trust, London, UK; ${ }^{4}$ University Hospitals Birmingham NHS Foundation Trust, Birmingham, UK; ${ }^{5}$ Newcastle Hospitals NHS Foundation Trust, Newcastle, UK

\subsection{6/gutjnl-2021-BASL.76}

Introduction Organ availability remains a barrier to successful management of patients with acute liver failure (ALF). Many patients have contra-indications to transplantation, leaving an ongoing unmet need for alternative and bridging therapies. High volume therapeutic plasma exchange (HV-TPE) has shown promising results in improving survival. In this multicentre retrospective analysis from the United Kingdom (UK) we present the largest cohort to date of patients undergoing therapeutic plasma exchange (TPE) for the treatment of ALF, using substantially smaller volume exchanges (LV-TPE) than previously studied.

Methods Consecutive patients admitted to 5 transplant centre intensive care units (ICU) and receiving TPE treatment for ALF between June 2013- July 2020 were included. Treatment with TPE was based on judgement of the treating clinicians. Clinical and biochemical parameters were recorded on admission and following the final TPE. Outcome measures included changes in the biochemical, physiological and organ function status, overall survival and transplant free survival.

Results 120 patients received TPE treatment for ALF. Median age was $38(30-52)$ and $59 \%(n=71)$ were female. The majority $(72.5 \%)$ had ALF due to paracetamol overdose and

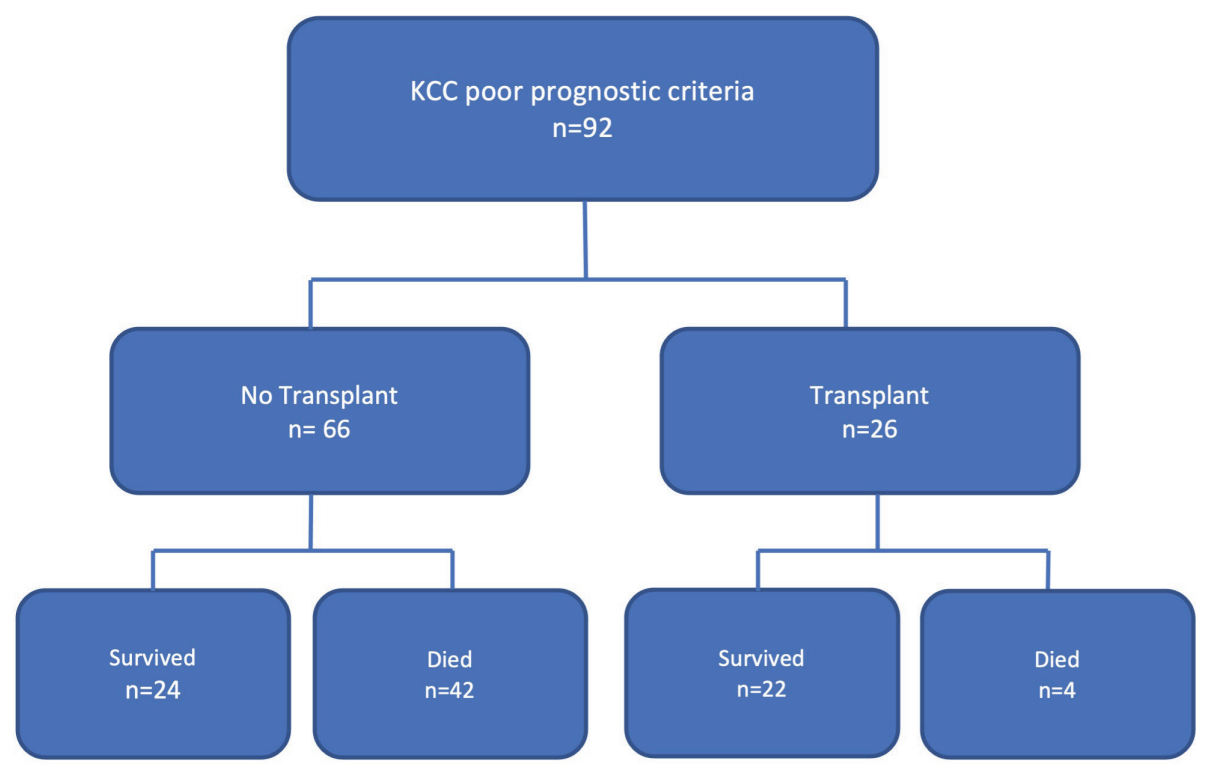

Abstract P067 Figure 1 


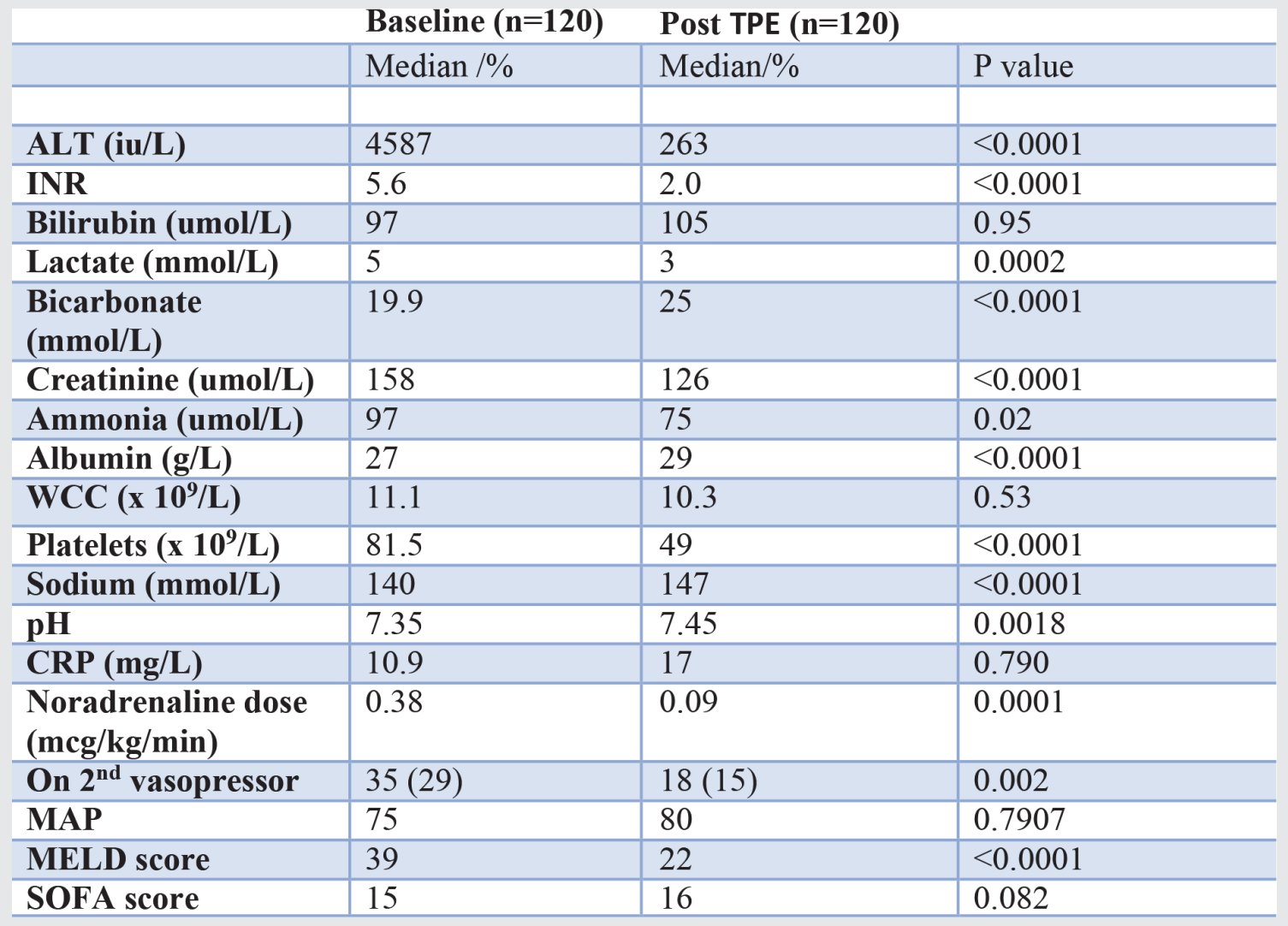

76.6\% of patients met Kings College Criteria (KCC) for poor prognosis. Median number of TPE sessions performed was 3 (IQR 2-3), with a median volume of 4 litres exchanged (IQR 3-5litres). Table 1 shows the changes in clinical variables pre and post TPE. Significant improvements $(p<0.05)$ were seen in the majority of clinical and biochemical variables. Notably there was a reduction in noradrenaline doses $(0.4 \mathrm{mcg} / \mathrm{kg} / \mathrm{min}$ to $0.09 \mathrm{mcg} / \mathrm{kg} / \mathrm{min}, \mathrm{p}=0.0001$ ) and in requirements for a second vasopressor agent $(29 \%$ to $15 \%, p=0.002)$. This benefit remained when subgroup analysis of patients fulfilling poor prognostic KCC was performed. Figure 1 shows in those meeting KCC overall survival was 50\%. Transplant free survival was $36 \%$; higher than seen in previous cohorts of patients fulfilling KCC and receiving standard medical treatment.

Conclusion LV TPE improves clinical variables of patients with ALF comparable with results seen in HV TPE. TPE remains a promising supportive therapy in creating a haemodynamic window of stability for transplant and as an alternative therapy for patients unsuitable for transplantation.

\section{P068 CASE FINDING FOR HEPATIC FIBROSIS IN AT-RISK POPULATIONS - ENHANCED LIVER FIBROSIS SCORE DETECTS MORE IN ALCOHOL THAN NONALCOHOLIC FATTY LIVER DISEASE}

${ }^{1}$ Alex Hinkson*, ${ }^{1}$ Jacob Feathers, ${ }^{1}$ Jason Punnamkuzhy, ${ }^{1}$ Firuza Dzhakhangirli, ${ }^{1,2}$ lan AC Rowe, ${ }^{1}$ Richard Parker. 'Leeds Teaching Hospitals NHS Trust, Leeds, UK; ${ }^{2}$ Leeds Institute for Data Analytics, University of Leeds, Leeds, UK

10.1136/gutjnl-2021-BASL.77
Background Nonalcoholic fatty liver disease (NAFLD) and Alcohol-related liver disease (ALD) are the most common causes of chronic liver disease in the UK. Early detection of fibrotic liver disease can allow intervention aiming to reduce progression. We report on results of case finding in at-risk groups in community settings to identify patients with advanced liver fibrosis.

Method Patients undergoing diabetic health checks at in primary care, or patients with high-risk alcohol intake in alcohol treatment services were tested for liver fibrosis using the enhanced liver fibrosis (ELF) test. Patients with ELF $<9.5$ were deemed at low risk of fibrosis and not examined further. Patients with ELF $>9.5$ were referred for liver stiffness measurement (LSM) by FibroscanTM and clinical review. $\mathrm{LSM}>15 \mathrm{kPa}$ was considered indicative of advanced disease.

Results A total of 1092 patients were included: 518 patients with diabetes and 574 patients with high-risk alcohol intake. In the diabetic cohort $54.6 \%$ had ELF $>9.5$, compared with $28.2 \%$ in the alcohol cohort. LSM was done in 246 patients with diabetes and 117 patients with high-risk alcohol intake. The median LSM result was $6.2 \mathrm{kPa}$ in the diabetic cohort and $8.9 \mathrm{kPa}$ in the alcohol cohort. In the diabetic cohort, $7.3 \%$ of LSM results were $>15 \mathrm{kPa}$, compared to $30.8 \%$ in the alcohol cohort. 438 patients had LFTs done within 3 months of ELF testing, 181 of these had ELF >9.5 and 96 (53\%) had a normal ALT.

Conclusion Case finding in persons with diabetes in primary care yielded relatively few positive cases. In contrast, over a quarter of cases with high-risk alcohol intake identified with blood tests had advanced disease. Importantly, ALT is normal in most patients with advanced disease confirming that 